OPEN ACCESS

Edited by:

Xiaowu Wang,

Chinese Academy of Agricultural

Sciences, China

Reviewed by:

Sachiko Narita Isobe,

Kazusa DNA Research Institute,

Japan

Jianfei Zhao,

University of Pennsylvania, USA

*Correspondence:

Qixiang Zhang

zqxbjfu@126.com

tThese authors have contributed equally to this work.

Specialty section:

This article was submitted to Plant Genetics and Genomics,

a section of the journal

Frontiers in Plant Science

Received: 04 June 2016 Accepted: 15 August 2016 Published: 31 August 2016

Citation:

Zhou Y, Xu Z, Zhao K, Yang W, Cheng $T$, Wang $J$ and Zhang $Q$ (2016) Genome-Wide Identification, Characterization and Expression Analysis of the TCP Gene Family

in Prunus mume.

Front. Plant Sci. 7:1301 doi: 10.3389/fpls.2016.01301

\section{Genome-Wide Identification, Characterization and Expression Analysis of the TCP Gene Family in Prunus mume}

\author{
Yuzhen Zhou ${ }^{1+}$, Zongda Xu ${ }^{1,2 t}$, Kai Zhao ${ }^{1}$, Weiru Yang ${ }^{1}$, Tangren Cheng ${ }^{1}$, Jia Wang ${ }^{1}$ and \\ Qixiang Zhang ${ }^{1 *}$
}

${ }^{1}$ Beijing Key Laboratory of Ornamental Plants Germplasm Innovation and Molecular Breeding, National Engineering Research Center for Floriculture, Beijing Laboratory of Urban and Rural Ecological Environment, Key Laboratory of Genetics and Breeding in Forest Trees and Ornamental Plants of Ministry of Education, School of Landscape Architecture, Beijing

Forestry University, Beijing, China, ${ }^{2}$ College of Forestry, Shandong Agricultural University, Tai'an, China

TCP proteins, belonging to a plant-specific transcription factors family, are known to have great functions in plant development, especially flower and leaf development. However, there is little information about this gene family in Prunus mume, which is widely cultivated in China as an ornamental and fruit tree. Here a genome-wide analysis of TCP genes was performed to explore their evolution in P. mume. Nineteen PmTCPs were identified and three of them contained putative miR319 target sites. Phylogenetic and comprehensive bioinformatics analyses of these genes revealed that different types of TCP genes had undergone different evolutionary processes and the genes in the same clade had similar chromosomal location, gene structure, and conserved domains. Expression analysis of these PmTCPs indicated that there were diverse expression patterns among different clades. Most TCP genes were predominantly expressed in flower, leaf, and stem, and showed high expression levels in the different stages of flower bud differentiation, especially in petal formation stage and gametophyte development. Genes in TCP-P subfamily had main roles in both flower development and gametophyte development. The CIN genes in double petal cultivars might have key roles in the formation of petal, while they were correlated with gametophyte development in the single petal cultivar. The CYC/TB1 type genes were highly detected in the formation of petal and pistil. The less-complex flower types of $P$. mume might result from the fact that there were only two CYC type genes present in $P$. mume and a lack of CYC2 genes to control the identity of flower types. These results lay the foundation for further study on the functions of TCP genes during flower development.

Keywords: Genome-wide, TCP genes, expression analysis, Prunus mume, flower development

\section{INTRODUCTION}

Prunus mume Sieb. et Zucc. (Rosaceae, Prunoideae) has been cultivated in China for over 3,000 years for its prominent ornamental and economic value. This species has acquired favorable ornamental characteristics with various types of flowers, colorful corollas, pleasing fragrance, and early blooming in the Prunus genus (Zhang et al., 2012; Xu Z. et al., 2014). The flower development plays a vital role in ornamental value. In P. mume, the studies of the MADS-box gene family and SBP-box gene family have been performed to gain insights into flower development 
(Xu Z. et al., 2014; Xu et al., 2015). Nevertheless, the molecular regulation of its flower development are still almost unknown. The TCP genes have been involved in different aspects of plant growth, including: flower development, flower symmetry, shoot branching, leaf development, leaf morphogenesis, leaf senescence, male and female gametophyte development, and circadian clock (Palatnik et al., 2003; Li et al., 2005; AguilarMartinez et al., 2007; Busch and Zachgo, 2007; Koyama et al., 2007, 2010; Poza-Carrion et al., 2007; Efroni et al., 2008; Schommer et al., 2008; Nag et al., 2009; Giraud et al., 2010; Sarvepalli and Nath, 2011; Yang et al., 2012). The genome-wide analysis of TCP gene in $P$. mume is important to learn more about the molecular mechanisms of flower development.

The TCP genes encode conserved sequences of about 60 amino acids designated as the TCP domain. This domain may be important for activation or repression of transcription and is involved in protein-protein interactions (Manassero et al., 2013). A basic helix-loop-helix (bHLH) structure formed from the TCP domain is quite different from the HLH structure which is a DNA-binding domain of the transcription factors both in plants and animals (Cubas et al., 1999). The name of TCP was derived from the four first finding members of the TCP gene family, Teosinte Branched 1 (TB1) from Zea mays, CYCLOIDEA (CYC) from Antirrhinum majus, and the two Oryza sativa genes, PROLIFERATING CELL FACTORS 1 and 2 (PCF1 and PCF2). So far, many TCP genes have been identified in various plant species. For example, there are 24 TCP genes in Arabidopsis, 28 TCP genes in O. sativa, 30 TCP genes in Solanum lycopersicum, 52 TCP genes in Malus domestica, and so on (Martin-Trillo and Cubas, 2010; Parapunova et al., 2014; Xu R. et al., 2014). Based on the differences of the TCP domains, the TCP proteins are divided into two subfamilies (Classes I and II). In the Class I, biochemical characterization of TCP proteins has been studied mainly in rice (O. sativa). PCF1 and PCF2 are identified as DNA-binding proteins that have the ability to bind specifically to PROLIFERATING CELL NUCLEAR ANTIGEN (PCNA) promoter (Kosugi and Ohashi, 1997). These genes have been suggested to promote plant proliferation and growth, based on their expression in meristematic tissues. Most single mutants in class I have mild or no phenotypic defects (Takeda et al., 2006; Herve et al., 2009). In contrast, the class II genes are proposed to have a role in preventing growth and proliferation by observing the single and multiple mutants in their phenotypes (Hubbard et al., 2002; Aguilar-Martinez et al., 2007; Kim et al., 2008; Martin-Trillo and Cubas, 2010). The Class II is further subdivided into two clades: CIN clade and CYC/TB1 clade. The CIN clade genes exemplified by CINCINNATA (CIN) of A. majus participate in lateral organ development, and the CYC/TB1 clade genes basically have functions in flower development or lateral shoot development (Martin-Trillo and Cubas, 2010). An R domain is acquired in most members of the CYC/TB1 clade, but little members of the CIN clade contain the R domain (Cubas et al., 1999). Because a glutamic acid-cysteine-glutamic acid stretch has been found between the TCP and R domains in the subset of CYC/TB1 genes, CYC/TB1 clade is also called ECE clade (Howarth and Donoghue, 2006). Phylogenetic analyses reveal that the CYC/TB1 clade can be classified into three types of genes which have expanded by duplication in the groups: CYC1, CYC2, and CYC3. In Arabidopsis, there are no CYC2 genes which have a primary role in the development of floral dorsoventral zygomorphy (asymmetry). Genetic studies in Arabidopsis also suggest that CYC1 genes have retained the TB1 function to control the shoot branching. The CYC3 includes Arabidopsis BRANCHED1, expressed both in flower primordia and branches, indicating the unclear functions in the flower development (Aguilar-Martinez et al., 2007; Poza-Carrion et al., 2007).

MicroRNAs are a kind of non-coding small RNAs that regulate gene expression by binding target mRNAs, which causes translational inhibition or cleavage. In the CIN clade, there are some microRNA miR319 target genes that have been analyzed so far. MiR159 and miR319 have a common ancestor and about 17 identical nucleotides. These miRNAs interact with each other and have overlapping roles in regulating floral development (Hong and Jackson, 2015). To identify networks controlled by TCPs or miR319, microarray analysis of transgenic plants with different levels of these transcription factors have been executed (Palatnik et al., 2003; Schommer et al., 2008, 2012, 2014). The expression levels of miR319-targeted TCP genes were decreased by miR319 activity, while other TCP genes, which lack miR319 target sites, were unaffected. An excess of cell expansion that generated a super compound organ in tomato (S. lycopersicum) or a crinkled simple leaf in Arabidopsis and A. majus was caused by low TCP activity or high levels of miR319 (Nath et al., 2003; Palatnik et al., 2003; Ori et al., 2007). Current evidences indicated that these TCP transcription factors were regulated by miR319 which guides them to cleavage. This pathway supported the importance of the control of plant growth and development.

In this study, we first identified 19 TCP genes in P. mume, and then carried on comprehensive bioinformatics analyses of phylogeny, gene structure, conserved domains, chromosomal location and miRNA target sites. Finally, transcriptome sequencing (RNA-seq) was employed to study the expression patterns of these genes in various organs. In order to clarify their functions in floral organ development, the real-time quantitative RT-PCR were also performed at different developmental stages of flower bud differentiation. This study presents the first genome-wide analysis of the TCP gene family in Prunus species. The results of this study provide the foundation for further functional analyses of TCP genes in P. mume and other Prunus species. These results will also broaden our insight into the roles of TCP genes in regulating flower development and flower organ determination.

\section{MATERIALS AND METHODS}

\section{Identification and Annotation of $P$. mume TCP Genes}

Prunus mume genome sequences were acquired from the $P$. mume genome database ${ }^{1}$. To identify TCP genes in P. mume, TCP domain HMM profile ${ }^{2}$ (PF03634) was used as a query

\footnotetext{
${ }^{1}$ http://prunusmumegenome.bjfu.edu.cn/

${ }^{2}$ http://pfam.Janelia.org/
} 
to perform HMMER searches against the $P$. mume genome database. As recommended by the HMMER user's guide, an $e$-value threshold of 0.1 was carried out in these searches (Finn et al., 2011). All obtained sequences were put in InterPro ${ }^{3}$ to assure the existence of the TCP domain. Based on the results from InterPro, the sequences which did not include the TCP domain were eliminated. The names of $P$. mume TCP genes were assigned according to their scores for complete sequences out of HMMER searches. TCP genes in Arabidopsis thaliana were downloaded from TAIR $^{4}$ using the identifiers reported by Martin-Trillo and Cubas (2010).

\section{Phylogenetic and Gene Structure Analysis}

To study the phylogenetic relationships between TCP genes in $P$. mume and other species, the protein sequences of the identified $P$. mume TCP genes, 24 A. thaliana TCP genes, a Teosinte Branched1 (TB1) from Z. mays, a CYCLOIDEA (CYC) from A. majus, and the PROLIFERATING CELL FACTORS 1 and 2 (PCF1 and PCF2) from O. sativa (Supplementary Data 3), were used to generate the phylogenetic tree. First, multiple sequence alignments were performed using Clustal X2.0 (Supplementary Figure 3) (Larkin et al., 2007). Subsequently, phylogenetic trees were constructed by MEGA7.1 with the Maximum-likelihood (ML) method. These analyses were performed with default parameters except the bootstrap analysis which was employed using 1,000 replicates. The genomic sequences and structural information of $P$. mume TCP genes were downloaded from the $P$. mume genome database (Supplementary Data 1 and 2). Diagrams of exon-intron structures were obtained using Gene Structure Display Server 2.0.

\section{Conserved Domains and Motif Analysis}

All protein sequences of PmTCPs were submitted to the MEME online tool ${ }^{6}$ to identify conserved motif and structural divergences. These sequences were analyzed in MEME with the following parameters: repetition number, any; maximum motif number, 20; maximum motif width, 60; minimum motif width, 6. To annotate the identified motifs, Pfam and SMART ${ }^{7}$ were used. Multiple sequence alignment was carried out with DNAMAN. Protein sequence logo was created by the Weblogo online tool ${ }^{8}$.

\section{Chromosomal Location and miR159/miR319 Target Site Prediction}

On the basis of positional information in the $P$. mume genome project ${ }^{9}$, locations of the TCP genes on the P. mume chromosomes were assessed using MapDraw and Photoshop

\footnotetext{
${ }^{3}$ http://www.ebi.ac.uk/interpro/

${ }^{4}$ https://www.arabidopsis.org/

${ }^{5}$ http://gsds.cbi.pku.edu.cn/

${ }^{6} \mathrm{http}: / /$ meme-suite.org/tools/meme

${ }^{7}$ http://smart.embl-heidelberg.de/

${ }^{8} \mathrm{http}: / /$ weblogo.threeplusone.com/

${ }^{9}$ http://prunusmumegenome.bjfu.edu.cn/
}

software. To predict miR159 and miR319 target sites, fulllength PmTCPs nucleotide sequences were analyzed using the psRNATarget online application ${ }^{10}$.

\section{Expression Analysis}

Transcriptome sequencing (RNA-seq) was performed to study expression profiles of PmTCPs. Total RNA was extracted from five organs of $P$. mume: roots, stems, leaves, flowers, and fruits. The sequencing and assembly was executed at the Beijing Genomics Institute. Genesis software was used to standardize the expression data and estimate hierarchical clustering (Sturn et al., 2002).

\section{Real-Time Quantitative RT-PCR}

The expressions of 19 PmTCPs in flower buds at different development stages were examined using real-time RT-PCR. The materials of the gene expression pattern analysis in different periods of flower bud development were taken from the bud of 'Jiang Mei,' 'Sanlun Yudie,' and 'Subai Taige.' Two samples of basic consistent appearance were taken in every 5-7 days. One was sampled to define the development stages of the flower bud using paraffin section method; the other one was quickly frozen in liquid nitrogen and used for the extraction of RNA. Total RNA was extracted from flower buds at eight development stages (S1, S2, S3, S4, S5, S6, S7, and S8) using Trizol reagent (Invitrogen, USA) according to the manufacturer's instructions. And potentially residual genomic DNA was removed from RNA samples using RNase-free DNase (Promega, USA). To synthesis fist-strand cDNA from $2 \mu \mathrm{g}$ total RNA, the TIANScript First Strand cDNA Synthesis Kit (Tiangen, China) was used following the manufacturer's instructions. Real-time RT-PCR was completed with PikoReal real-time PCR system (Thermo Fisher Scientific, Germany) and reactions were performed in a $10 \mu \mathrm{l}$ volume including $0.5 \mu \mathrm{l}$ of $\mathrm{cDNA}, 200 \mathrm{nM}$ of each primer (Supplementary Table 1) and $5 \mu \mathrm{l}$ of SYBR Premix ExTaq II (Takara, China). The reactions were completed in the following conditions: $30 \mathrm{~s}$ at $95^{\circ} \mathrm{C}, 40$ cycles of $5 \mathrm{~s}$ at $95^{\circ} \mathrm{C}$ and $30 \mathrm{~s}$ at $60^{\circ} \mathrm{C}, 30 \mathrm{~s}$ at $60^{\circ} \mathrm{C}$, end in $20^{\circ} \mathrm{C}$ and the temperature of melting curve was set from 60 to $95^{\circ} \mathrm{C}$ rising at $0.2^{\circ} \mathrm{C} / \mathrm{s}$. The amplification efficiency of each primer pair was investigated by melting curve analysis. All experiments were performed with three biological duplications, and each duplication was repeated in triplicate. With the protein phosphatase 2A (PP2A1) gene of $P$. mume as the reference gene (Wang et al., 2014a), the relative expression levels were calculated with the $2^{-\Delta \Delta \mathrm{Ct}}$ method.

\section{RESULTS}

\section{Identification and Chromosomal Location of TCP Genes in P. mume}

To identify the TCP genes in P. mume, HMMER searches were employed to search against the protein database of $P$. mume using TCP domain HMM profile PF03634 as the search query. Through the search, a total of 19 sequences were obtained. The Interpro

\footnotetext{
${ }^{10}$ http://plantgrn.noble.org/psRNATarget/?function
} 
analysis revealed that each of the 19 protein sequences contained the TCP domain, indicating that there were at least 19 TCP genes in the $P$. mume genome (Table 1). In these genes, four TCP genes were found possessing the R domain. As shown in Table 1, these TCP proteins varied in their length, molecular weight, and theoretical isoelectric point. The protein length ranged from 229 to 516 amino acids, the molecular weight from 24.93 to $55.70 \mathrm{kDa}$ and the theoretical isoelectric point varied from 5.38 to $9.51 \mathrm{pH}$.

Based on the genomic data, we located 14 PmTCPs on chromosomes (Figure 1), but five genes were found on the anchored scaffolds. The TCP genes were unevenly distributed over the $P$. mume genome. No genes were located on Chr 1, Chr 6, and Chr 8. Whereas, two genes were located on Chr 5. Furthermore, the Chr 2, Chr 3, Chr 4, and Chr 7 chromosomes each had three genes. Two P. mume TCP genes, PmTCP03, and $P m$ TCP06, were located in duplicated regions. None of the TCP genes was tandemly duplicated.

\section{Phylogenetic and Gene Structure Analysis}

According to multiple sequence alignments of 19 PmTCPs, 24 AtTCPs, 2 OsPCFs, $1 \mathrm{AmCIN}, 1 \mathrm{AmCYC}$, and $1 \mathrm{ZmTB1}$, two individual subfamilies (Classes I and II genes) were generated using MEGA7.1 with the ML method. Class I is named TCP-P class or PCF class, and Class II is named TCP-C class. The class II genes were further divided into two lineages: CYC/TB1 and CIN. There were 10 PmTCPs in class I and 9 PmTCPs in class II (Figure 2). The OsPCF1 and OsPCF2 were found in TCP-P subfamily. Genes of $P$. mume in both class I and class II virtually had counterparts in Arabidopsis. These genes first grouped with their homologous genes in Arabidopsis. In regard to class II, three of them were CYC/TB1 type genes, and the others were CIN type genes. $A m C Y C$ and $Z m T B 1$ were found in CYC/TB1 group. In the CYC subgroup, there were only two P. mume genes (PmTCP01 and PmTCP02). PmTCP01 was gathered with AtTCP12 identified as CYC3 type gene and PmTCP02 was gathered with AtTCP01 identified as CYC1 type gene. The other gene, PmTCP07, was grouped with AtTCP18 identified as BRC1 which was the closest homolog of TB1. In CIN subgroup, $A m C I N$ were found grouped with six other PmTCPs. The phylogenetic tree suggested that some genes may have undergone species-specific evolutionary processes.

Introns, and especially UTR introns, in TCP genes may influence their expression levels (Chung et al., 2006). Based on the genome sequences and corresponding coding sequences of TCP genes in $P$. mume, we found that no TCP-C type genes, except PmTCP02 and PmTCP11, contained introns (Figure 3). The PmTCP02 intron was in the $3^{\prime}$ UTR, revealing that it might have a regulation role in the gene expression. Sequences of TCP-P genes contained more introns than those of TCP$\mathrm{C}$ genes in $P$. mume. In the TCP-P genes, the numbers of introns ranged from zero to four. Six of them had no introns, three had an intron, and one had four introns. In conclusion, the exon-intron structure showed itself to be highly simple in P. mume.

\section{Conserved Domains and Motif Analysis}

The conserved domains of TCP protein were examined by Interpro searches and aligned by ClustalX 2.0. Alignment of the TCP domains as well as the dendrogram suggested that the 19 TCP proteins in $P$. mume could be divided into two subfamilies. These PmTCPs all contained the TCP domain which is involved in dimerization and DNA binding. The TCP domain

TABLE 1 | Inventory and characteristics of the TCP genes identified in Prunus Mume.

\begin{tabular}{|c|c|c|c|c|c|c|c|}
\hline Name & Gene ID & Locus & Strand & Length ${ }^{a}$ & $M W(k D a)^{b}$ & $\mathrm{pl}^{\mathrm{c}}$ & Introns \\
\hline PmTCP01 & Pm029002 & scaffold205:763920:765428 & - & 502 & 55.70 & 8.16 & 0 \\
\hline PmTCP02 & Pm015499 & Pa4:19881270:19882614 & - & 417 & 46.79 & 8.48 & 0 \\
\hline PmTCPO3 & Pm016133 & Pa4:23296564:23298211 & - & 280 & 29.58 & 9.33 & 1 \\
\hline PmTCPO4 & Pm006518 & Pa2:17274760:17276031 & + & 423 & 44.71 & 7.00 & 0 \\
\hline PmTCP05 & Pm003922 & Pa2:1205292:1206842 & - & 516 & 53.42 & 7.21 & 0 \\
\hline PmTCP06 & Pm007054 & Pa2:21100313:21101122 & + & 269 & 28.72 & 9.51 & 0 \\
\hline PmTCPO7 & Pm030202 & scaffold475:648769:650115 & + & 448 & 51.01 & 6.42 & 0 \\
\hline PmTCP08 & Pm018100 & Pa5:16193275:16194165 & - & 296 & 31.68 & 7.15 & 0 \\
\hline PmTCPO9 & Pm010440 & Pa3:4466020:4467519 & - & 499 & 54.19 & 7.29 & 0 \\
\hline PmTCP10 & Pm023748 & Pa7:7860942:7862067 & - & 332 & 34.39 & 5.38 & 1 \\
\hline PmTCP11 & Pm029788 & scaffold327:681062:682143 & + & 335 & 37.09 & 7.30 & 1 \\
\hline PmTCP12 & Pm019399 & Pa5:23705247:23706395 & - & 382 & 39.69 & 6.25 & 0 \\
\hline PmTCP13 & Pm010118 & Pa3:2653873:2654736 & - & 287 & 32.61 & 6.42 & 0 \\
\hline PmTCP14 & Pm030138 & scaffold475:312338:313027 & - & 229 & 24.93 & 5.98 & 0 \\
\hline PmTCP15 & Pm011369 & Pa3:10626210:10627529 & - & 439 & 47.64 & 6.79 & 0 \\
\hline PmTCP16 & Pm016049 & Pa4:22847778:22848908 & + & 376 & 41.40 & 6.59 & 0 \\
\hline PmTCP17 & Pm024946 & Pa7:15188102:15189265 & - & 387 & 42.93 & 6.95 & 0 \\
\hline PmTCP18 & Pm024934 & Pa7:15092655:15095383 & + & 376 & 40.36 & 7.46 & 1 \\
\hline PmTCP19 & Pm028040 & scaffold103:259784:262394 & + & 463 & 50.63 & 6.04 & 4 \\
\hline
\end{tabular}

a The length of P. mume TCP proteins. ${ }^{b}$ Molecular weight. ${ }^{C}$ Theoretical isoelectric point. 


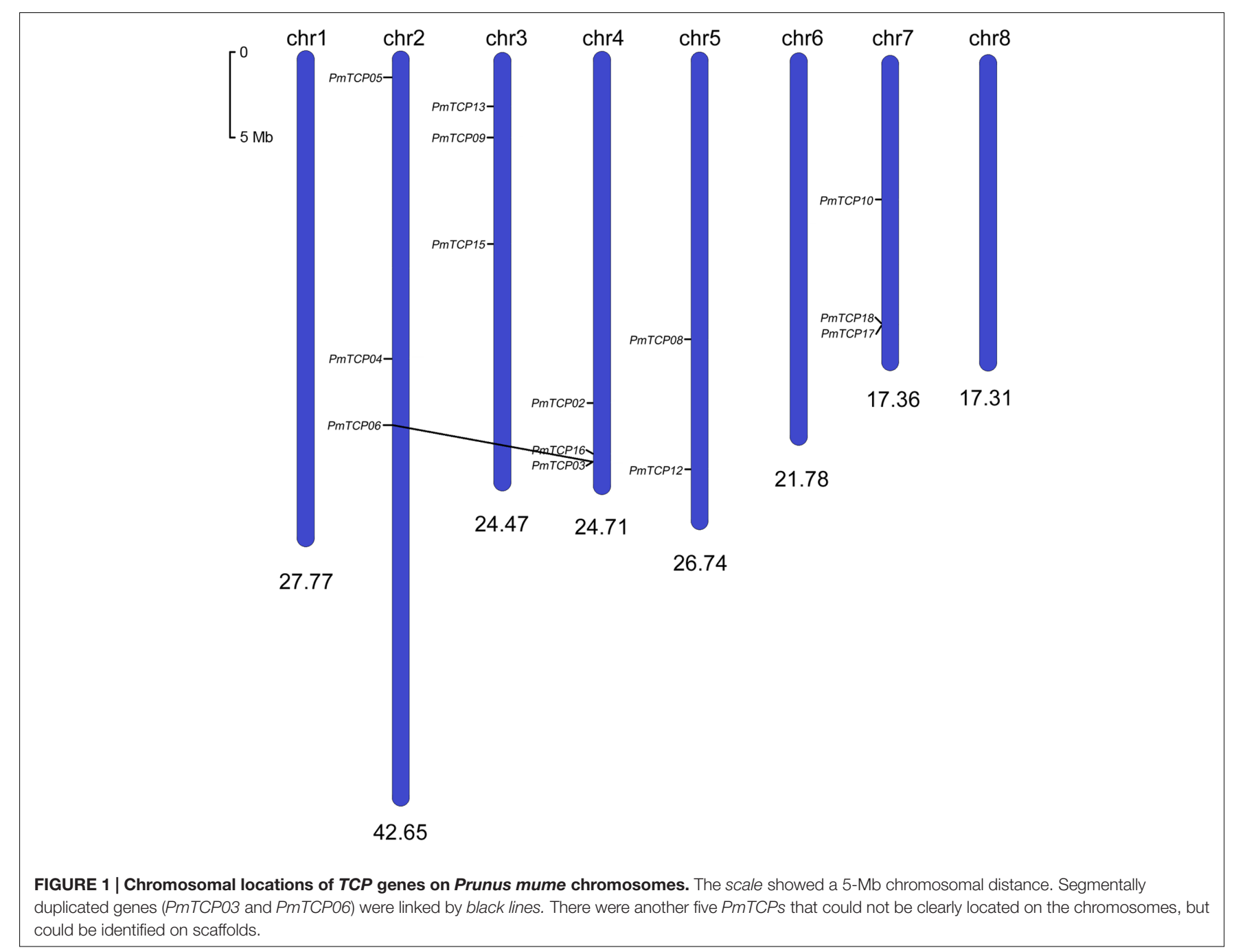

in P. mume consisted of 55 or 59 amino acids and participated in a basic-Helix-Loop-Helix (bHLH) structure (Figure 4A). The hydrophobic residues required for helix-helix interactions in bHLH proteins were conserved in the TCP domain (Manassero et al., 2013). Within the TCP domain, there were several putative residues involved in DNA binding located in the basic region and several putative hydrophobic residues located in Helixes I and II (Figure 4A). In the basic region, the TCP-C type proteins contained a 4-amino-acid insertion. The compositions of the loop, Helixes I and II, were quite different between TCP-P and TCP-C. There were four PmTCPs containing an R domain (Figure 4B). These PmTCPs were all classified as TCP-C type genes. Among them, PmTCP01, PmTCP02, and PmTCP07 were CYC/TB1 type genes, and PmTCP09 was a CIN type gene.

To identify conserved motif and evaluate structural divergences, MEME online tool was used to analyze the motif compositions of the 19 TCP proteins in P. mume. Among the PmTCPs, the number of motifs varied from three to eight. Three motifs, motif 1 , motif 2 , and motif 3 , were specific to the TCP-domain. As shown in Figure 5, motifs 1 and 2 were present in all sequences. Motif 3 was unique to TCP-P subfamily. Outside the TCP domain, there were other conserved motifs specific to clades, such as motif 6 identified as the $\mathrm{R}$ motif. Members of the same clade usually had similar motif composition. The duplicated genes, PmTCP03 and PmTCP06 belonging to TCP-P subfamily, showed the same motif composition and distribution. Motifs 3, 4, 7, 9, 10, 11, and 14 were unique to TCP-P subfamily, while motifs $6,8,12,13,15$, and 18 were restricted to TCP-C subfamily. Some motifs, such as motif 5 , were shared in two different classes.

\section{MiR159/miR319 Target Site Prediction}

In P. mume, three miR159 family members (Pmu-miR159a-c) and only one miR319 (Pmu-miR319) had been identified (Wang et al., 2014b). A result of one miR159 target site (PmTCP9) was obtained from analysis in the psRNATarget online software with the full-length of PmTCPs nucleotide sequences and PmumiR159b sequence. However, three putative miR319-targeted TCP genes (PmTCP9, PmTCP11, and PmTCP15) were identified based on the published miRNAs (Supplementary Table S2) in the psRNATarget online application. These three miR319 target sites were all located in the coding regions (Figure 6A). And all 


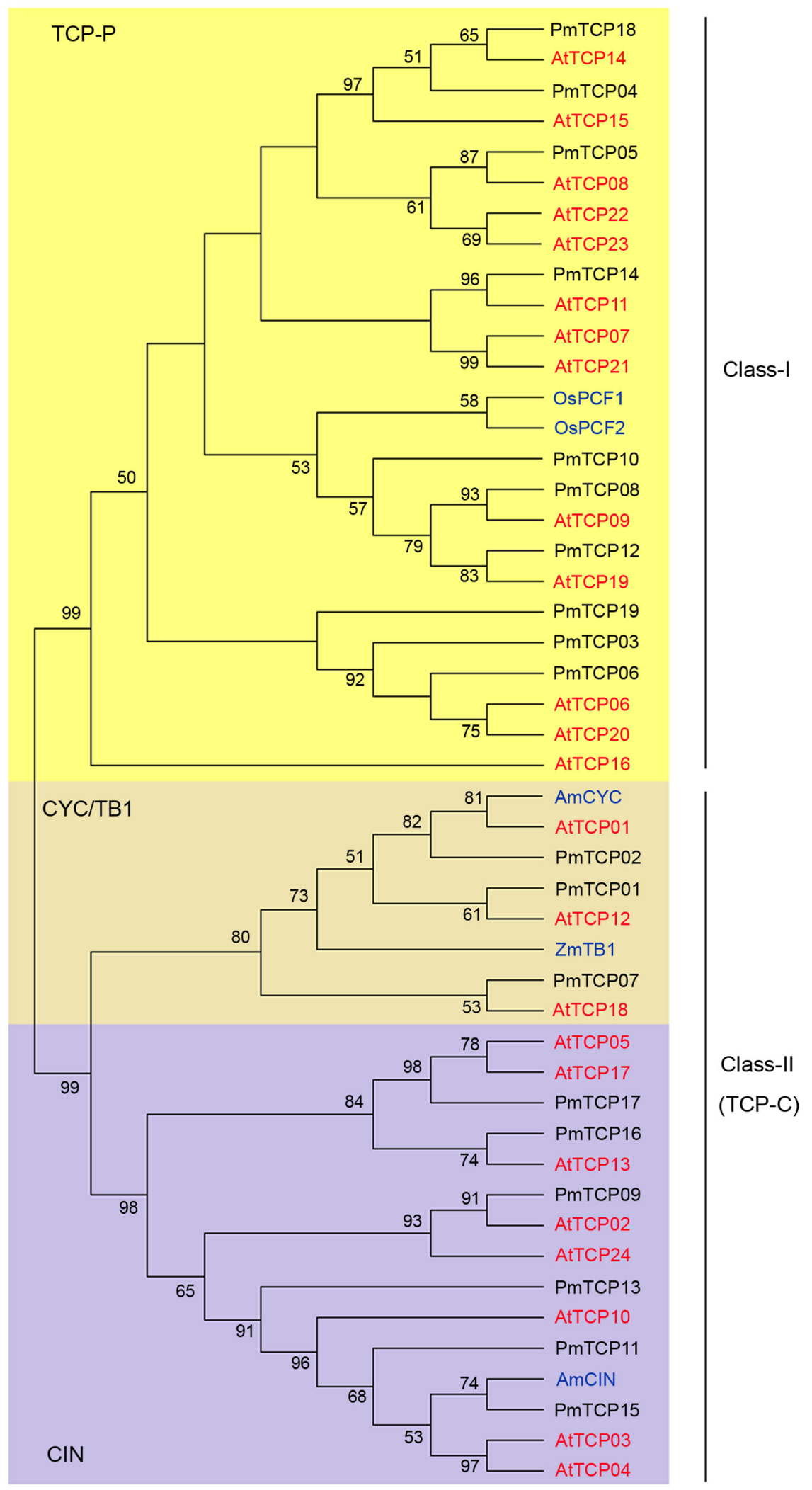

FIGURE 2 | Phylogenetic tree of TCP genes. The tree was constructed using MEGA7.1 with Maximum-likelihood (ML) method. Multiple sequence alignment of the TCP proteins was applied using clustal X2.0. Numbers above branches represented bootstrap value, and the bootstrap values which below 50 would not be shown. 


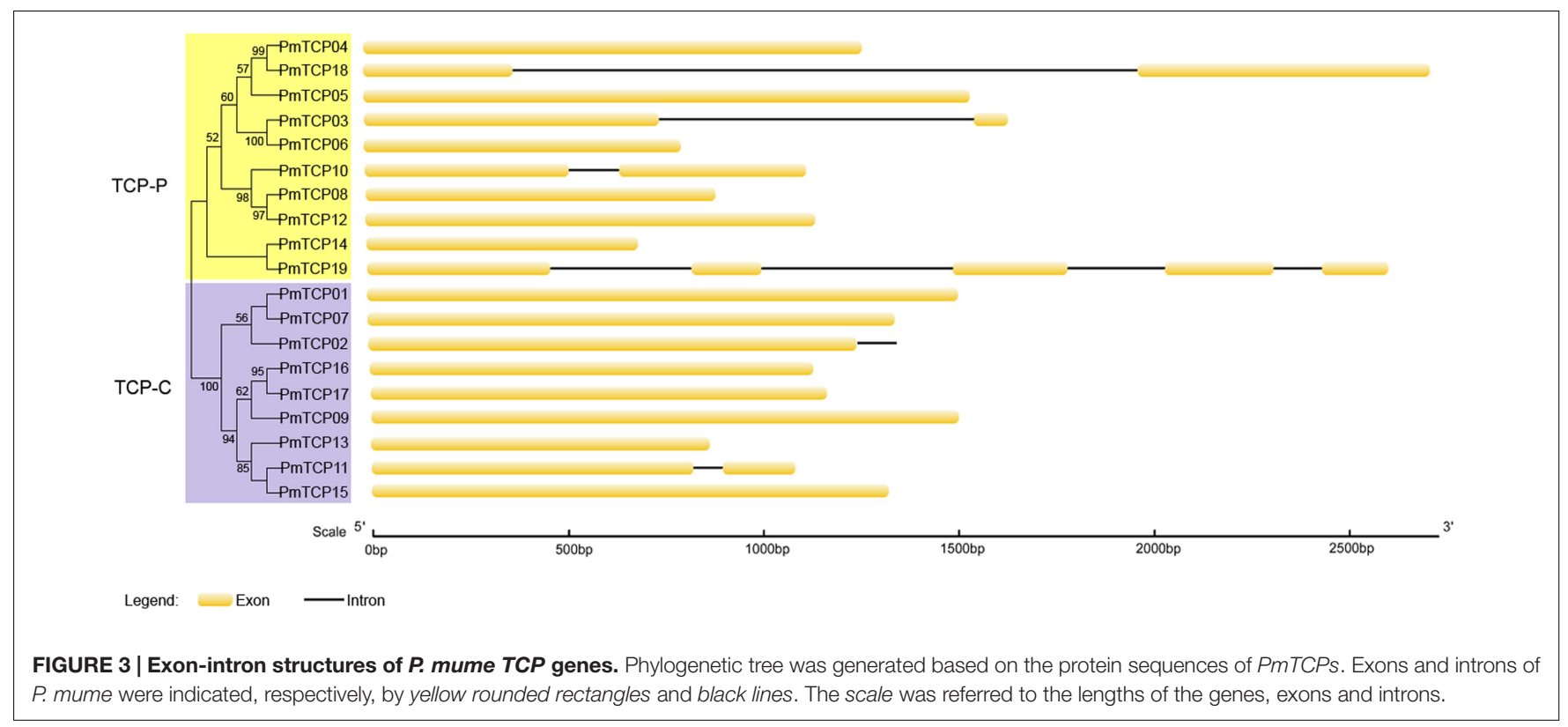

miR319 targeted genes belonged to CIN clade. Similarly, there were five TCP genes containing miR319 binding sites (AtTCP2, AtTCP3, AtTCP4, AtTCP10, and AtTCP24) in Arabidopsis, and all of them were CIN family members. This phenomenon revealed that the miR319 target sites were retained during the evolution and diversification of plants.

The members of miR319 and miR159 were very similar but encoded by different genes and precursors (Schommer et al., 2012). Sometimes they were grouped in one family, whereas others considered them separately (Meyers et al., 2008). In $P$. mume, the Pmu-miR159c sequence was virtually identical to miR319 aside from one differing base located at position 1 (Figure 6B). Thus, we considered Pmu-miR159c as a member of miR319 family in P. mume. Multiple sequence alignments of the reverse miR319 sequence and three $P$. mume TCP genes showed that the PmTCPs containing sequences are complementary to the miR319 sequence (Figure 6B). Though a mismatch of miR319 and mRNA came up to six bases, the regulation of the expression of TCP transcription factor through miR319 was considered to be feasible (Schommer et al., 2012).

\section{Expression Profiles of $P$. mume TCP Genes in Five Organs}

According to previous studies, the TCP genes had key roles in different aspects of plant development. In this study, the next-generation transcriptome sequencing was performed to investigate the expression profiles of $P$. mume TCP genes in different organs and determine their function in organ development. As shown in Figure 7, the expression levels of TCP genes varied in five organs; flowers, leaves, and stems had the most numbers of expressed genes and the highest expression level. The expression of two genes could not be discovered in leaves and roots, both of which genes belong to TCP-C subfamily. The other 15 genes were expressed in all five organs, indicating that these genes might play roles in the development of diverse organs.

The expression patterns of the different type genes were shown in Figure 7A. In general, the TCP-P type genes had wider expression domains and higher expression levels than did TCP$\mathrm{C}$ type genes. All TCP-P genes were expressed in all five organs. This result implied that the genes in TCP-P subfamily played multiple developmental roles in different organs. However, most of TCP-C type genes showed organ-specific expression modes. In TCP-C subfamily, the CIN genes usually had specific prevailingexpression organs, but some of them were expressed in five organs. Furthermore, the genes belonging to CYC/TB1 clade had developmental functions in specific organs. All CYC/TB1 genes were either mildly or not at all expressed in roots, implying these genes might not participate in root development.

A hierarchical clustering was shown in Figure $7 \mathbf{B}$ and performed to do a further analysis of the organ development in which TCP genes could be involved. The TCP genes in P. mume could be grouped into two clusters according to their expression patterns. Cluster 1 contained the genes whose transcripts were detected in all five organs; but the genes in Cluster 2 did not always have the same expression profiles. The genes belonging to cluster 1 could be divided into two expression groups. One gene formed one group, exhibiting high expression levels in flower, fruit, leaf, and stem. The other group was composed of eight genes and exhibited even expression levels in all organs. Cluster 2 included four expression groups. The first group presented high expression levels in all organs except roots. There were three genes in the second group showing high expression levels in stem and flower. The genes in the third group were strongly expressed in flower and leaf, but their transcripts could not be detected at all in root and stem. Differing from these three groups, the fourth group did not show an organ-specific expression pattern and had relatively stable expression levels in all five organs. The analysis of hierarchical clustering in TCP genes showed that some 


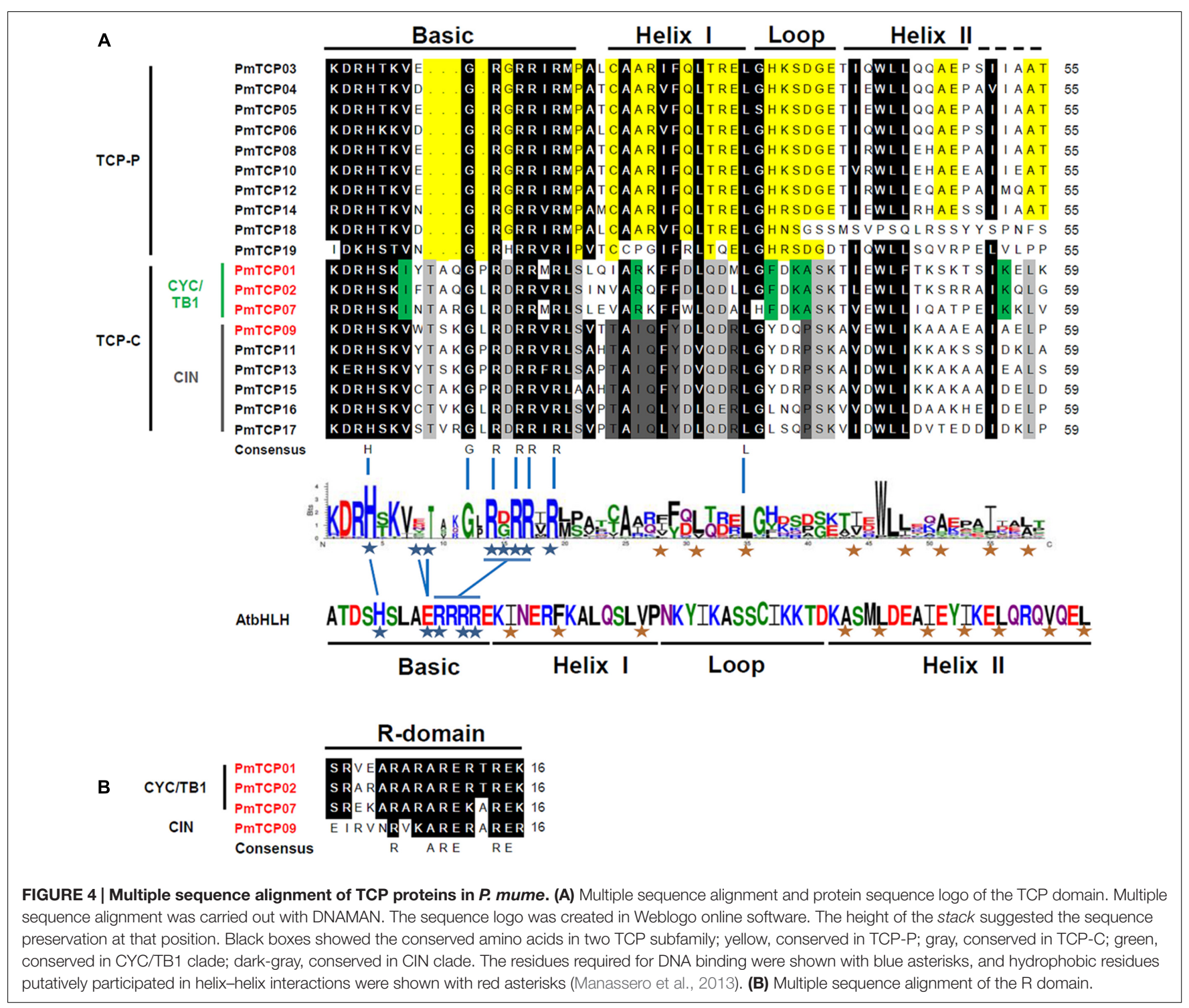

groups from different clusters had similar expression profiles. For example, two clusters had a group of genes which were expressed in all organs.

\section{Expression Analysis of PmTCPs during Flower Bud Development and in Different Flower Type Cultivars}

To elucidate their roles in flower development, the quantitative RT-PCR were conducted to confirm the relative expression levels of 19 PmTCPs during flower bud development. Based on the paraffin section analyses (Supplementary Figure 1), the flower bud differentiation of $P$. mume was divided into eight stages (S1S8): undifferentiation (S1), flower primordium formation (S2), sepal initiation (S3), petal initiation (S4), stamen initiation (S5), pistil initiation (S6), ovule development (S7), anther development (S8). Samples were taken from the flower buds of 'Jiang Mei', 'Sanlun Yudie', and 'Subai Taige' (Supplementary Figure 2).
The flower type of 'Jiang Mei' is single, while 'Sanlun Yudie' and 'Subai Taige' are double. 'Subai Taige' has a variant pistil which might form a defective flower. All 19 PmTCPs were detected during flower bud differentiation in three $P$. mume cultivars, suggesting that most of them play key roles in flower development.

In 'Jiang Mei' (Figure 8A), different types of TCP genes were quite variously expressed in different stages of flower development. The CYC1 type gene, PmTCP02, was significantly expressed in petal initiation. The transcript of PmTCP07 was highly detected in the stages of stamen initiation and anther development. This result suggests that PmTCP07, a homolog of TB1, might participate in regulating stamen development. The CIN type genes were strongly expressed in pistil initiation, ovule and anther development. The TCP-P subfamily, according to the expression, could be divided into two groups. The genes in group one were almost uniformly expressed in eight stages, but some of them seemed to have an unconspicuous function in flower 


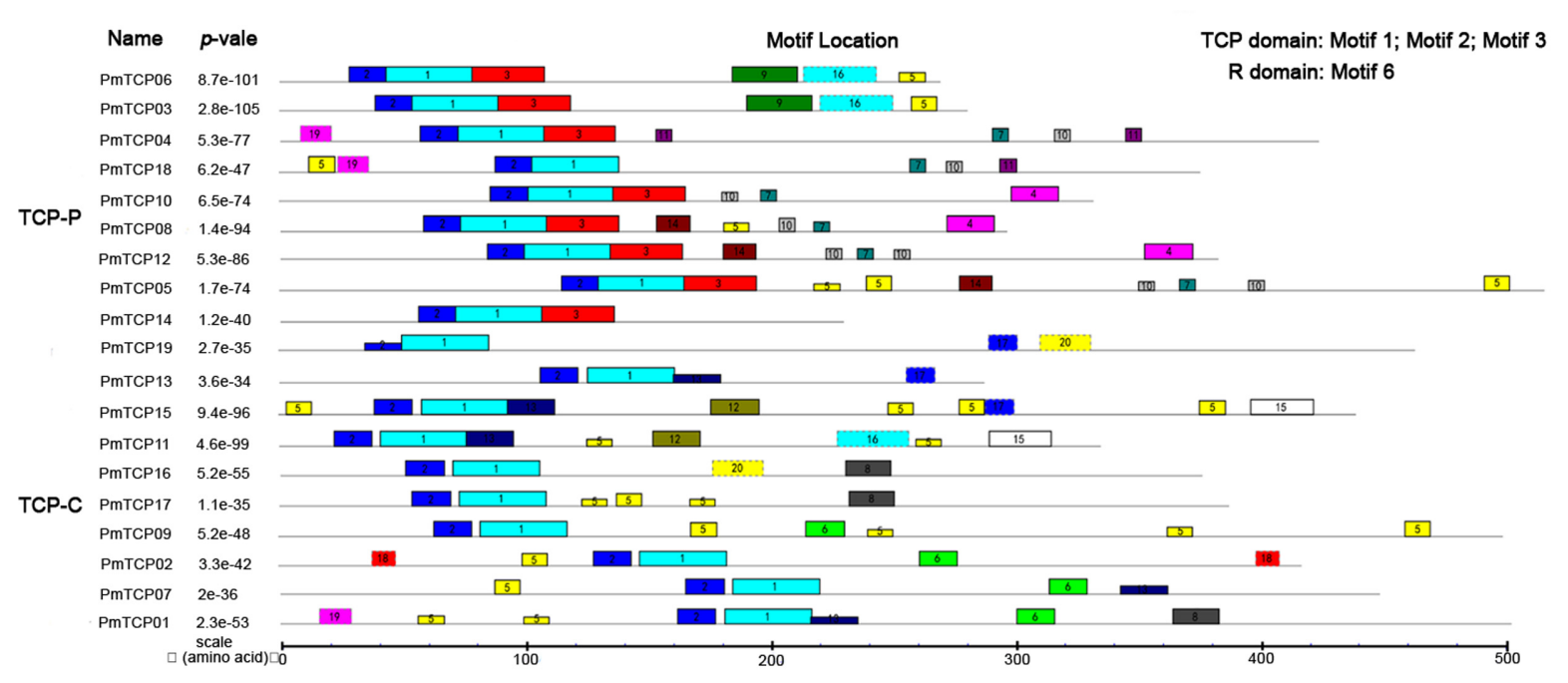

FIGURE 5 | Motif distribution of TCP genes in P. mume. Twenty motifs were indicated using MEME online tool. A colored block with a number was consistent with each motif (color figure online). The lengths and positions of motifs in the protein sequences were identified by the lengths and positions of the blocks.

A

\begin{tabular}{|l|l|l|l|}
\hline 5'UTR & TCP & & 3'UTR \\
\hline \multicolumn{2}{|c|}{ Intact mRNA } & \\
\hline & $\begin{array}{c}\text { miR319-directed } \\
\text { cleaved product }\end{array}$ \\
\hline \hline
\end{tabular}

B

PmTCP9

PmTCP11

PmTCP15

miR319

Pmu-miR159c

PmTCP9

Pmu-miR159b
5' $A G G G G G A C C C \cup \cup C A G \cup C C A A \cup 3^{\prime}$ 3' $A C C \cup C G A G G G A A G \cup \cup A G G \cup \cup C 5^{\prime}$

FIGURE 6 | The miR319 binding sites of TCP gene in P. mume. (A) The TCP gene structure showed the coding region (blue), the TCP domain (green), the miR319 binding site (red). The putative miR319-directed cleaved product was represented by the short fragment. (B) Alignment of miR319 complementary sequences with $P$. mume TCP genes. Mismatches, G-U wobbles and the different nucleotide between Pmu-miR159c and miR319 were represented, respectively, by Red, blue, and black (bold). The direction of the red arrows was indicated the miR319 recognition site in the TCP genes. In the red rectangular region was the changed nucleotide of miR319 shifted relative to miR159.

primordium formation and sepal initiation. Others had specific expression in ovule and anther development, or petal initiation.

Compared to 'Jiang Mei', there were also many TCP genes with different functions expressed in flower development in 'Sanlun Yudie' (Figure 8B). The CYC/TB1 type genes, PmTCP01, PmTCP02, and PmTCP07, were predominantly expressed in flower primordium formation, sepal initiation and pistil initiation. There were other genes in the TCP-P subfamily which showed expression primarily in flower primordium formation and pistil initiation as well. The transcripts of CIN type genes were mostly detected in pistil initiation and anther development. Briefly, in 'Sanlun Yudie', the majority of TCP genes in $P$. mume were highly expressed in flower primordium formation, pistil initiation and anther development. 
A
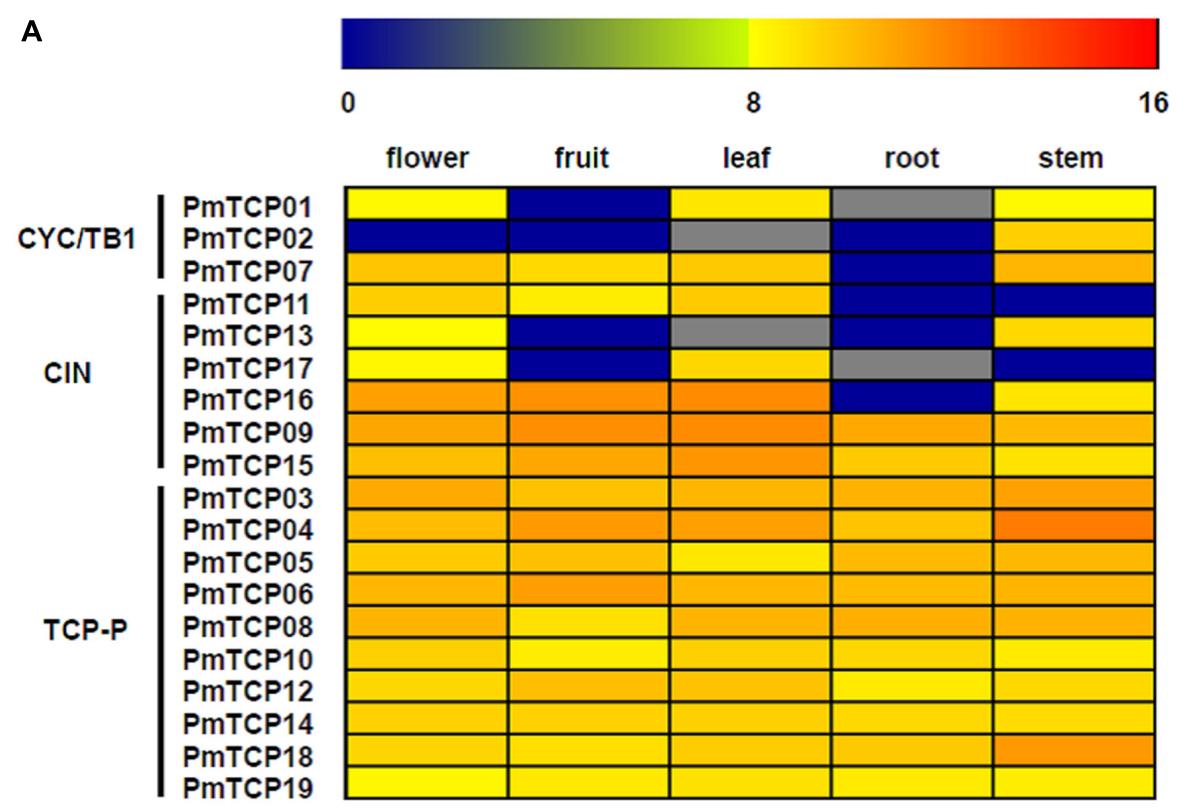

B

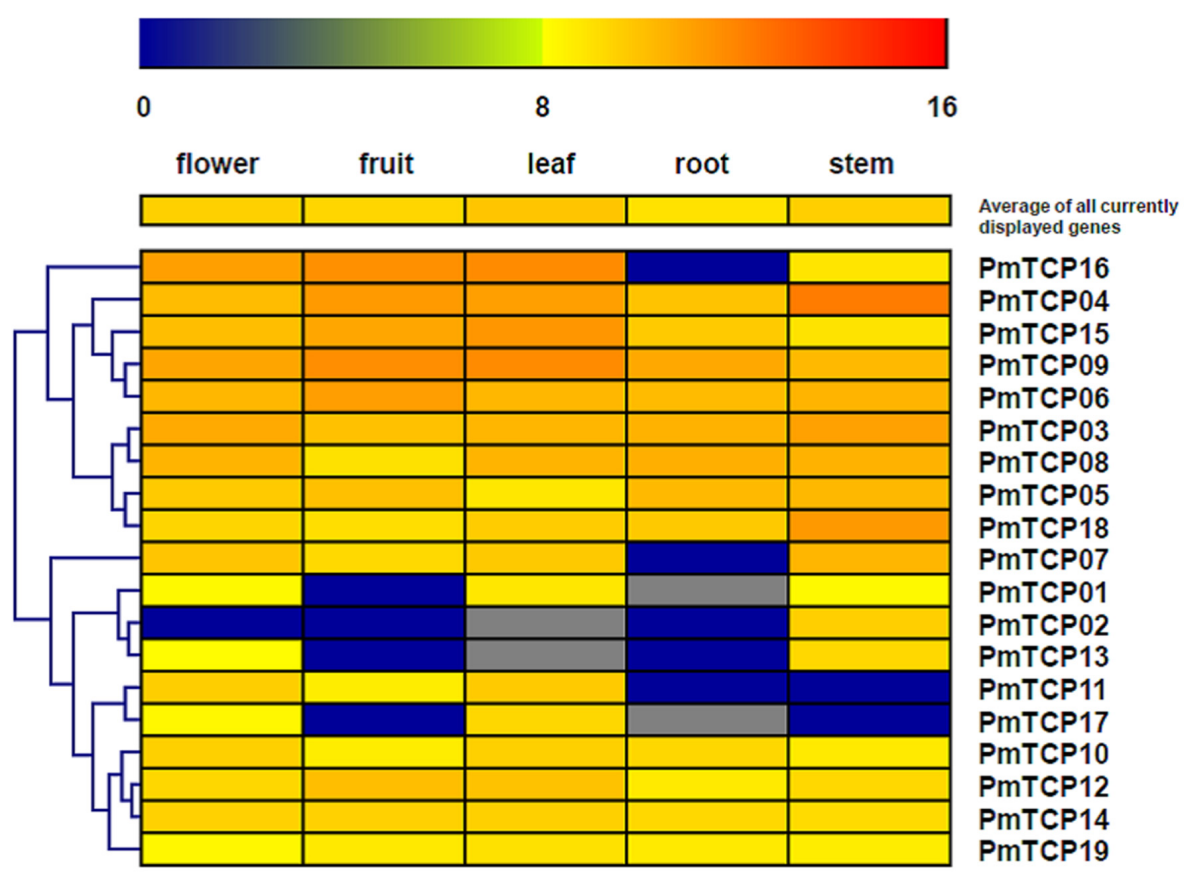

FIGURE 7 | Expression patterns of $\boldsymbol{P}$ mume TCP genes in five tissues. Transcriptome sequencing (RNA-seq) was performed to investigate expression profiles of TCP genes. The RPKM-normalized log2-transformed counts were represented by the color scale. Blue suggests low expression levels and red suggests high levels. (A) Expression profiles related to subfamilies; (B) hierarchical-clustering analysis of gene expression profiles.

In 'Subai Taige, the expression patterns of TCP genes were distinct with the other two cultivars (Figure 8C). In CYC/TB1 clade, the genes showed high transcript levels in petal initiation and pistil initiation. The CIN type genes were divided into two groups according to their quite diverse expression profiles. One group of genes had high expression levels in flower primordium formation or petal initiation. The others were highly expressed in pistil initiation or anther development. In TCP-P subfamily, there were two kinds of expression patterns as well. Some genes had high expression levels in flower primordium formation and 
A
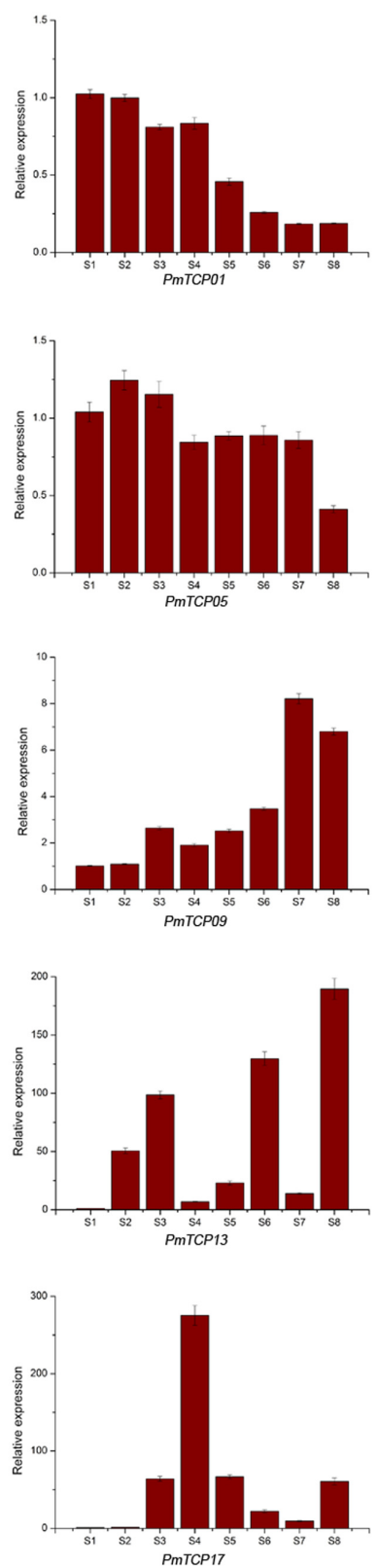
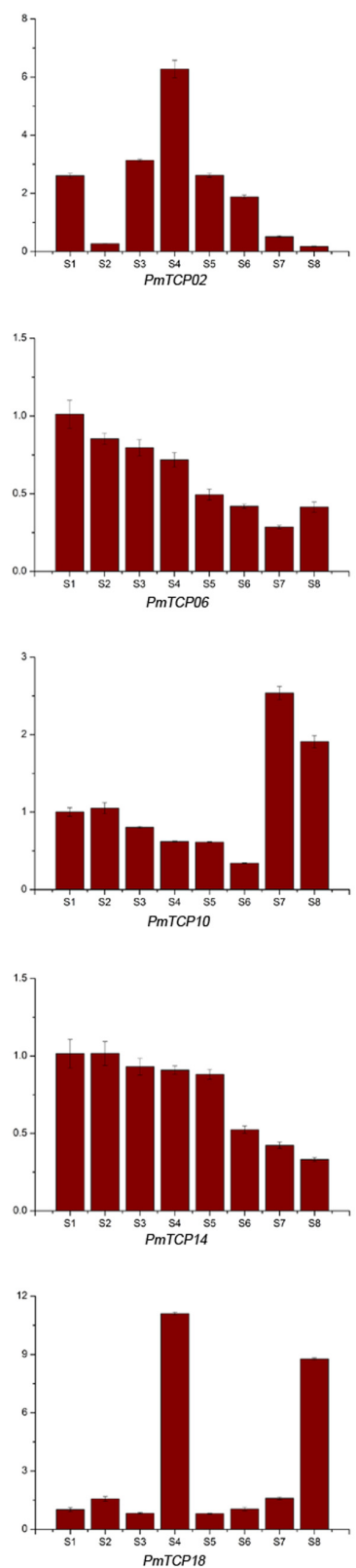
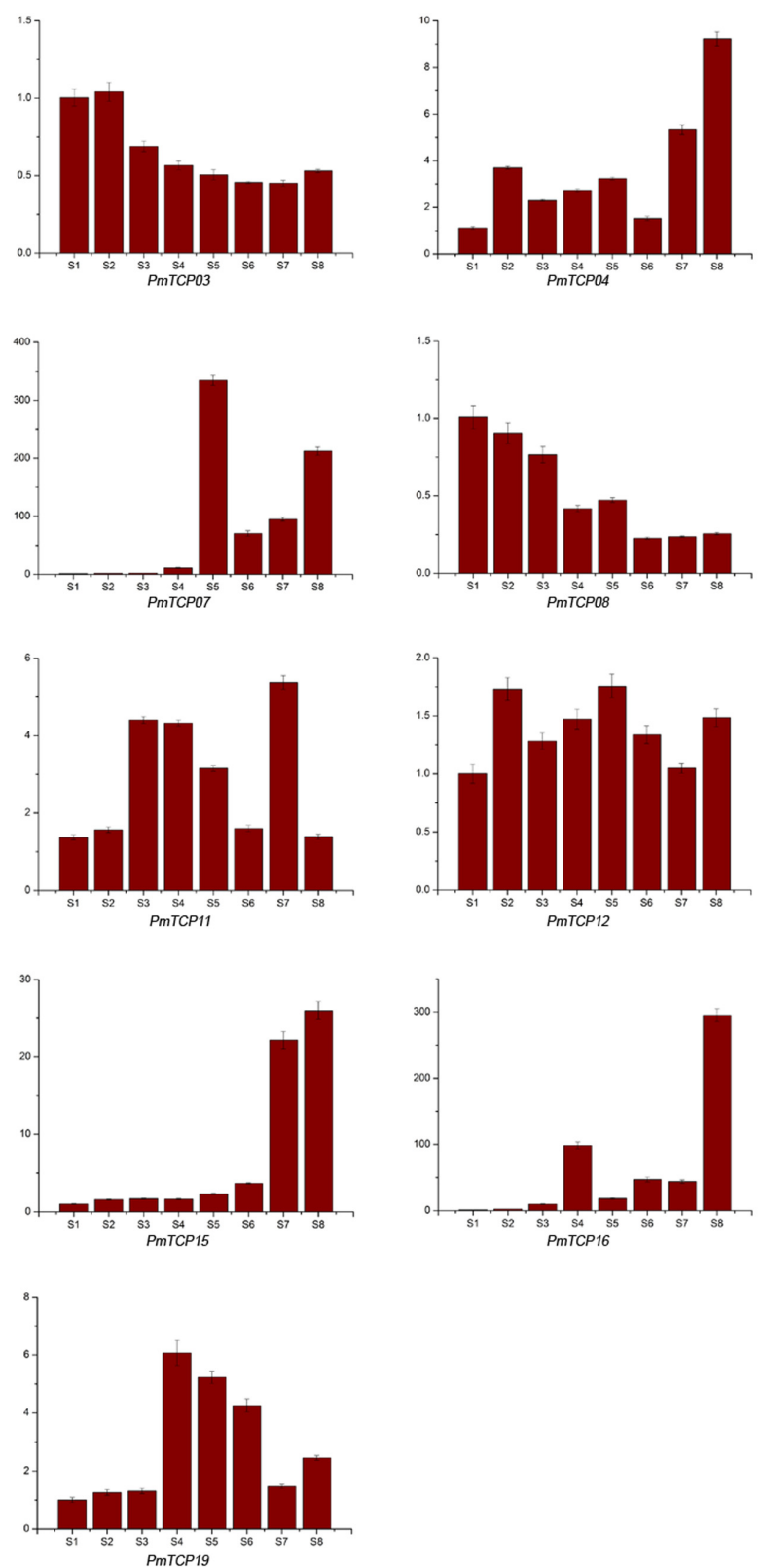

FIGURE 8 | Continued

sepal initiation. The others were specifically expressed in pistil initiation.

\section{DISCUSSION}

\section{TCP Genes in $P$. mume and Their} Evolution

The TCP transcription factors are ancient plant-specific proteins. Although they have not been found in the unicellular algae, they are present in pluricellular green algae, moss, ferns, and lycophyte (Martin-Trillo and Cubas, 2010). There are five to six TCP genes in these plants. Due to the evolution, duplication and diversification of TCP genes, the gymnosperms and angiosperms have larger TCP families comprising tens of members. In this study, we identified 19 TCP genes in P. mume genome.

The analysis of the phylogenetic tree between Arabidopsis and $P$. mume supported the earlier described division of TCP genes into two subfamilies. According to that division, PmTCPs were 
B
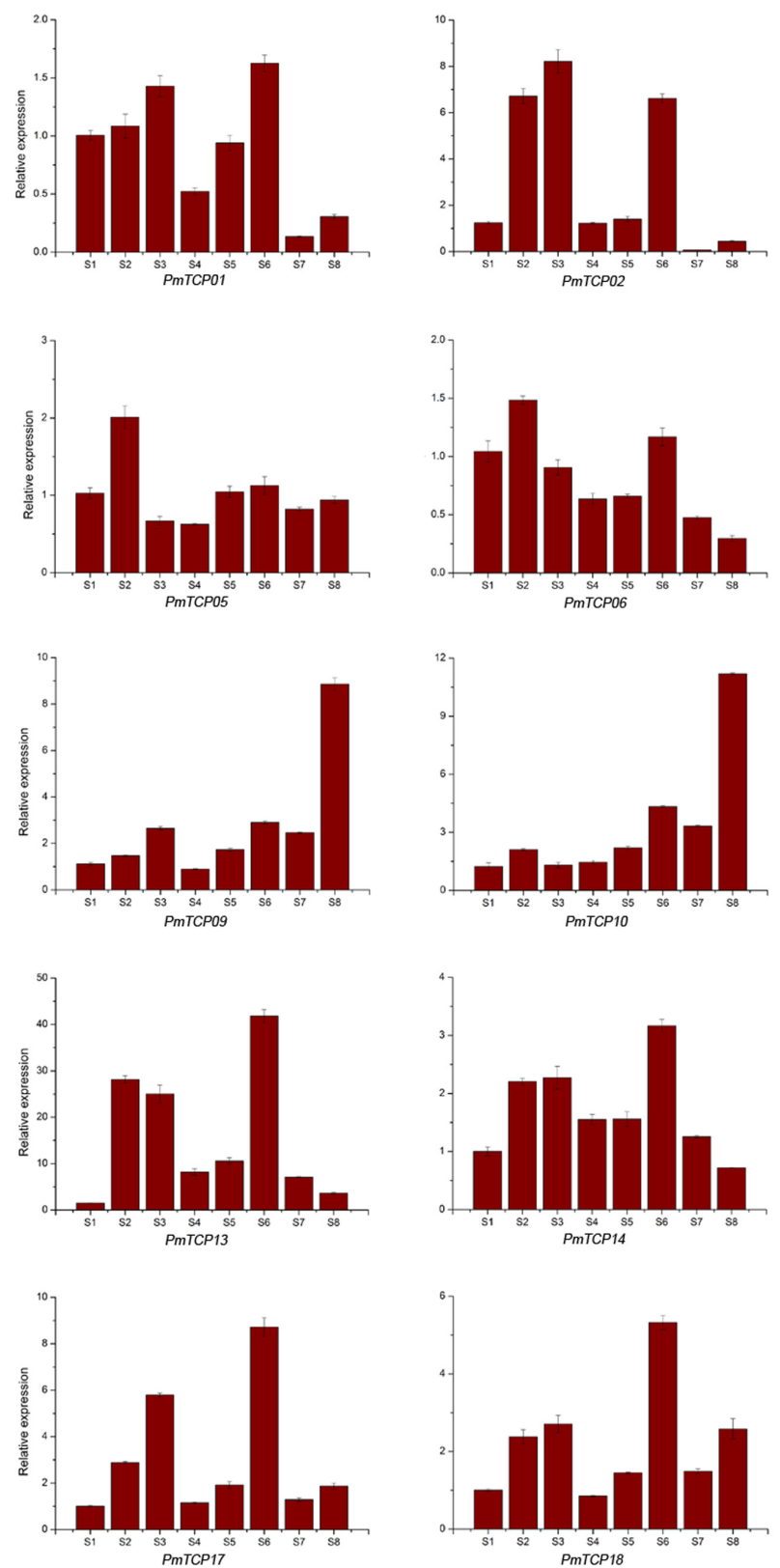
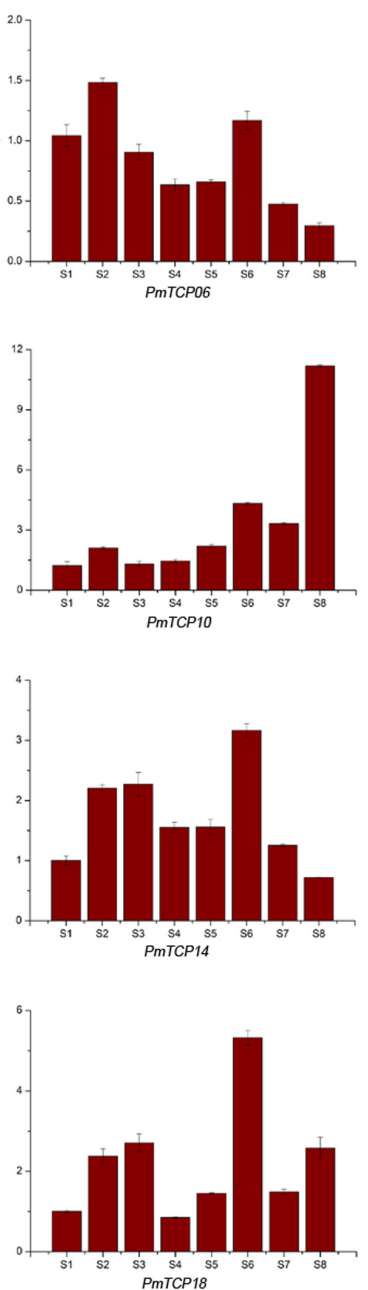
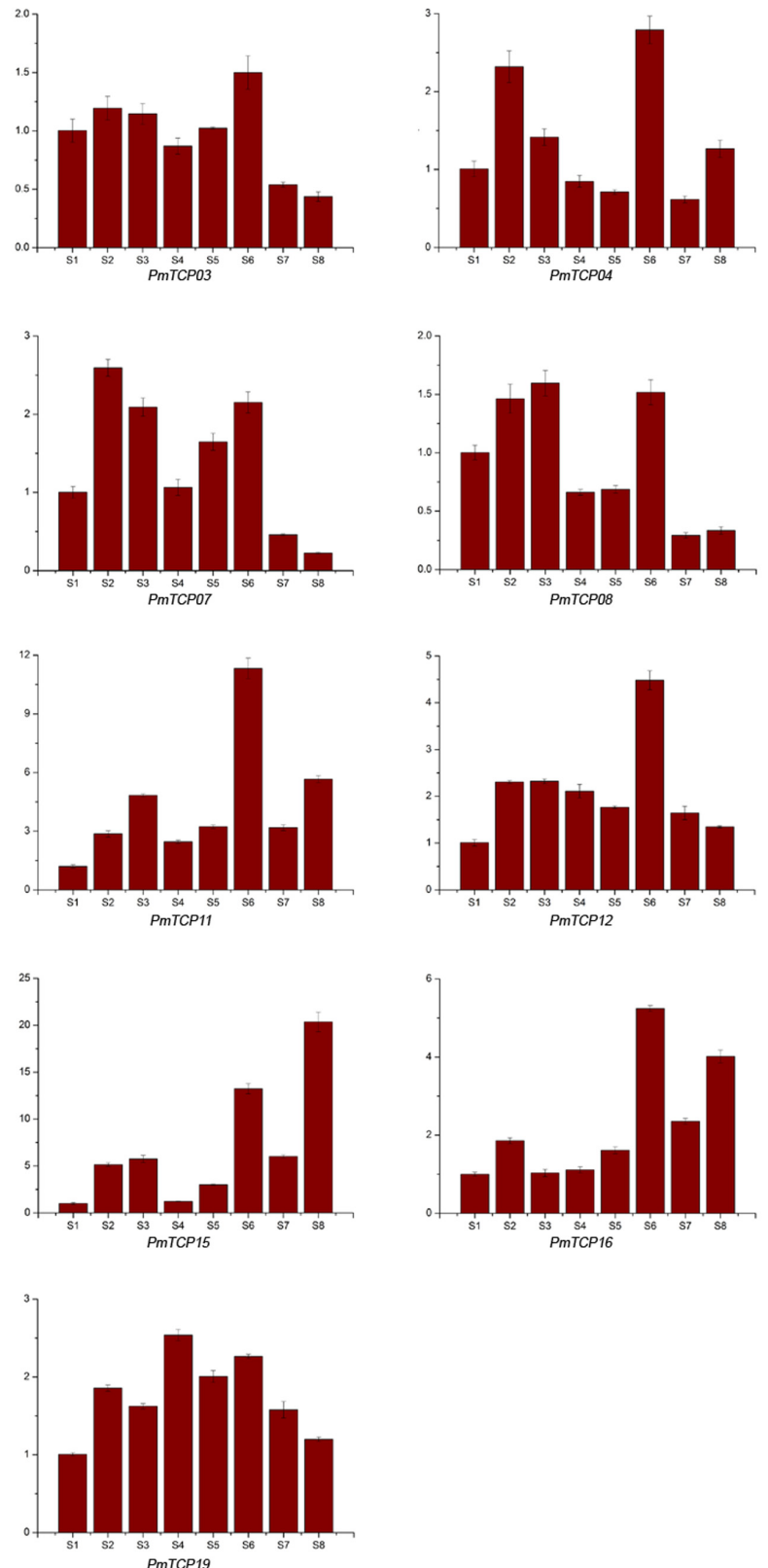

FIGURE 8 | Continued

classified as TCP-P genes and TCP-C genes. Two types of TCP genes were distributed regularly on five chromosomes (Figure 1). Two TCP-P type genes, PmTCP03 and PmTCP06, were located in duplicated regions. The phylogenetic analysis indicated that the lower number of $P$. mume TCP genes was the result of fewer gene duplication events in P. mume. Only for AtTCP16 can a close homolog not be found in $P$. mume. A similar phenomena was also observed in tomato (Parapunova et al., 2014). The alignment of the TCP domains in P. mume showed a similar result to that of the dendrogram. High similarity of proteins within the same clade suggested that gene duplications had occurred after the split of the two classes. Compared with the analysis of the conserved domains, the exon-intron structures of $P$. mume TCP genes exhibitted high conservatism. Generally, genes within a clade shared a similar gene structure, chromosomal location, and motif distribution. However, there were variations in gene structure, motif composition, and miR319/159 target site location among the different clades. Therefore, the classification and evolution of P. mume TCP genes might be related to their structural divergence and diversification. 


\section{C}
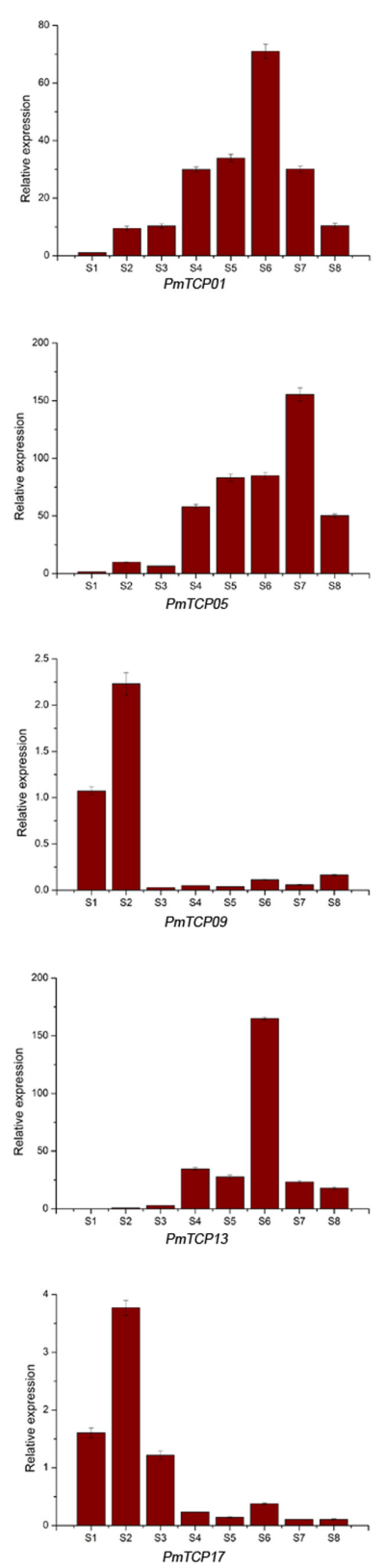
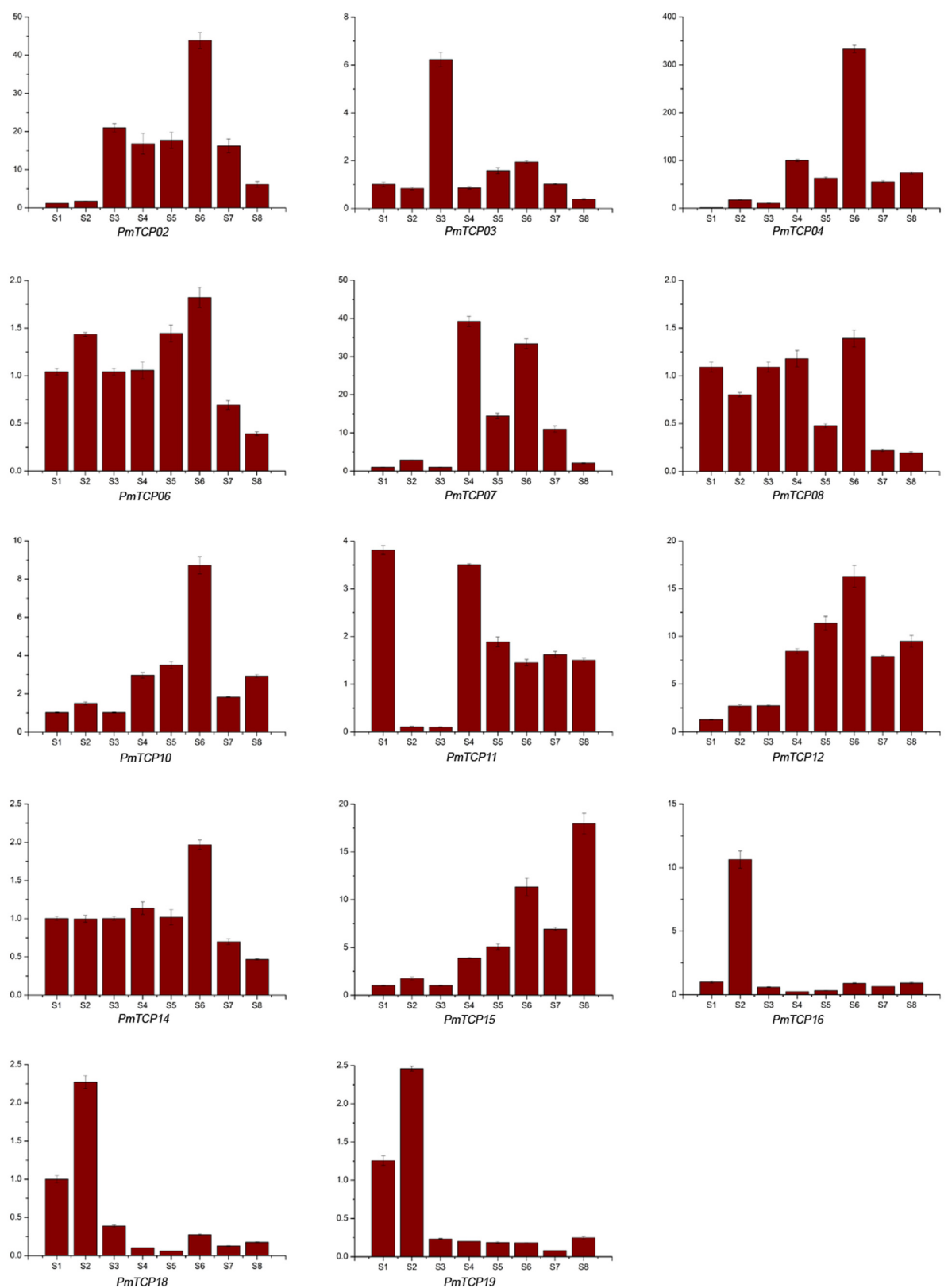

FIGURE 8 | Expression profiles of TCP genes in three $P$. mume cultivars (A-C successively were 'Jiang Mei', 'Sanlun Yudie,' and 'Subai Taige') during flower bud development. S1-S8 indicate development stages of flower bud. The expression levels of the TCP genes were gained by quantitative real-time RT-PCR. The reference gene was the Protein Phosphatase 2A (PP2A1) gene of P. mume. All real-time RT-PCR experiments were employed with three biological duplications, and each duplication was repeated in triplicate. The standard deviation of the results of three technical replicates was shown by the error bars.

\section{Expression Patterns of $P$. mume TCP Genes}

The expression profile of a gene is always relative to its function (Xu et al., 2015). In this study, we elucidated the expression patterns of 19 PmTCPs in five organs by RNA-seq and detailed expression profiles in flower development using real-time RTPCR. The results showed that these genes had distinct expression profiles in diverse organs, suggesting that TCP genes in P. mume might be correlated with the development of various organs. We observed a divergence in expression patterns between two subfamilies of genes. In TCP-C subfamily, more than two-thirds of genes had a specific-organ expression. However, all TCP-P genes and some CIN genes were detected in all organs and predominantly expressed in flower, leaf, and stem. The duplicated 
genes (PmTCP03 and PmTCP06), belonging to TCP-P subfamily, showed a similar expression profile. The expression patterns of some genes were similar to their orthologs in Arabidopsis. For instance, eight CIN type genes in Arabidopsis (AtTCP2, AtTCP3, AtTCP4, AtTCP5, AtTCP10, AtTCP13, AtTCP17, and AtTCP24) exhibited high transcript levels in leaf and were responsible for the regulation of leaf growth (Palatnik et al., 2003; Ori et al., 2007). Similarly, the CIN type genes in P. mume except PmTCP13 were expressed strongly in leaf. These genes were also highly expressed in flower. According to RNA-seq analyses, the flower contained almost all PmTCPs transcripts, indicating that TCP genes in $P$. mume had function in floral organ development.

The PmTCPs were expressed during flower bud differentiation, implying that some $P$. mume TCP genes play roles in the different stages of flower bud development. The TCP-C type genes, whether in CYC/TB1 clade or in CIN clade, were involved in the development of petal and pistil. For all three cultivars, the CYC/TB1 type genes were highly expressed in petal initiation or pistil initiation, indicating that they controlled the formation of petal and pistil. In Antirrhinum, CYC and DICH have been found to regulate dorsoventral asymmetry and are highly expressed in developing stamens (Gaudin et al., 2000; Galego and Almeida, 2002). The genes in CYC2 subclade take part in the formation of floral zygomorphy (Yang et al., 2012). Furthermore, CYC genes may be related to a change of petal size in Arabidopsis (Costa et al., 2005). The less-complex flower types of $P$. mume might result from the fact that there were only two CYC type genes present in P. mume and a lack of CYC2 genes to control the identity of flower types.

In the single petal cultivar, 'Jiang Mei,' the CIN type genes were highly expressed in the stages of pistil initiation, ovule and anther development, implying that these genes were correlated with gametophyte development. In other species, such as Arabidopsis, AtTCP4 which belongs to CIN clade was found to be involved in pollen development (Sarvepalli and Nath, 2011). In the other two cultivars which were all double type, the CIN type genes were predominantly expressed in flower primordium formation and petal initiation. This expression pattern indicated that the CIN genes in double petal cultivars might have more important roles in the formation of petal, which is quite different to their roles in the single petal cultivar.

Similarly, the expression patterns of the TCP-P subfamily genes were different in two flower types of $P$. mume. For the single petal cultivar, the TCP-P type genes might play a key role in flower primordium formation or petal initiation. For the double petal cultivars, most of these genes were strongly expressed in flower primordium formation and pistil initiation. During pistil initiation development, the inner whorl of petals of 'Sanlun Yudie' were continual to form a double flower (online resource 3). These results revealed that $P m T C P s$ in double petal cultivars might participate in the regulation of the petal formation for their predominant expression in the stages of petal initiation and pistil initiation. In conclusion, genes in TCP-P subfamily have a main role in flower development and gametophyte development of P. mume. In Arabidopsis, AtTCP11 and AtTCP16 participate in gametogenesis as well (Takeda et al., 2006; Viola et al., 2011).
In 'Subai Taige,' some genes expression was continued in order to form the petals in pistil primordium for its variable pistils. Contrasting with the other two cultivars, TCP genes in 'Subai Taige' had particularly specific expression patterns in flower primordium formation, but were hardly expressed in ovule and anther development. The variant of pistil which was replaced by a defective flower in 'Subai Taige' might be due to the lack of high expression levels of some PmTCPs in ovule and anther development, such as PmTCP09.

\section{Different Expression Patterns of miR319-Targeted and -Non-targeted TCP Genes in $P$. mume}

Previous studies revealed that over half of the miRNA targets are transcription factors, for instance: TCP, MYB, SBP, AP2, and so on (Rhoades et al., 2002; Aukerman and Sakai, 2003; Martin-Trillo and Cubas, 2010; Xu et al., 2015). Concerning P. mume TCP genes, three of them (PmTCP09, PmTCP11, and PmTCP15) contained putative miR319 target sites and one of them had a putative miR159 target site. They were all located in the coding region. In P. mume, the miR319 had two differences with respect to miR159: an additional base at their $5^{\prime}$ end but one less base at $3^{\prime}$ end; and a change of $\mathrm{C}$ for $\mathrm{U}$ at position 6 so that miR319 sequences were shifted one nucleotide relative to miR159 (Figure 4B). The same was true for Arabidopsis. MiR319 and miR159 in Arabidopsis had very similar sequences but regulated different genes. MiR159 did not target the TCPs due to specific sequence requirements (Palatnik et al., 2007). Though miR159 cannot bind TCP transcripts, it has an overlapping role with miR319 in controlling floral development and interacts with each other (Hong and Jackson, 2015). Current studies indicate that some TCP transcription factors were regulated by miR319. The TCP mRNA fragments are generated by a miR319a-guided cleavage and confirmed in vivo (Palatnik et al., 2003). In P. mume, the miR319-directed cleaved products were predicted in miR319regulated TCPs (Figure 6A).

The miR319s are involved in multiple aspects of plant development such as flower, leaf and gametophyte development (Hong and Jackson, 2015). The miR319-targeted genes in P. mume, except PmTCP11, were ubiquitously expressed in the five organs examined. All three genes were predominantly expressed in flower, leaf, and fruit, and PmTCP09 was particularly significant. PmTCP09 had both miR319 and miR159 target sites.

In plants, the miR319 family participates in the control of flower development with the miR319-TCP model. The defects in petal and stamen development were observed in a loss-offunction miR319 mutant (Nag et al., 2009). Similar to miR159, overexpression of miR319 causes stamen defects and male sterility (Palatnik et al., 2007). During flower bud differentiation, the transcripts that contain a miR319 target site as well as miR319-non-targeted TCP genes were all detected in three $P$. mume cultivars. These three miR319 target genes were all involved in petal formation and gametophyte development. In different flower types of $P$. mume, the main phenotype differences occurred in petal initiation, pistil initiation and ovule development. Accordingly, miR319-TCPs seemed to have 
an important role in the formation of the different flower types in $P$. mume. Nevertheless, the development of flower and gametophyte were controlled not only by simple miR319targeted genes, but also by different types of PmTCPs which lacked a miR319 target site. For instance, PmTCP04 and PmTCP10 which were TCP-P type genes and PmTCP13 which was a CIN type gene were all highly expressed in pistil initiation, ovule and anther development. Therefore, TCP genes in P. mume might have a coefficient and interactional relationship in the development of flower and gametophyte.

\section{CONCLUSION}

In this study, we first executed the genome-wide analyses of TCP gene family in $P$. mume. Based on phylogenetic analysis, we then concluded that the lower number of $P$. mume TCP genes was the result of less gene duplication events in $P$. mume. Only one segmental duplication event was found in the $P$. mume TCP family. Among the same type of TCP genes, the gene structure exhibitted high conservatism. These findings set a foundation for future research on the evolution of TCP genes in plants. The expression analyses showed that most of PmTCPs were involved in flower development, leaf development and stem development. Otherwise, almost all PmTCPs played key roles in the different stages of flower bud development, especially in petal formation and gametophyte development. Compared to the expression patterns of the miR319-targeted and non-targeted genes, TCP genes in $P$. mume might have a coefficient and interactional relationship in flower development. Perhaps the most interesting questions which arise from this research are what kinds of

\section{REFERENCES}

Aguilar-Martinez, J. A., Poza-Carrion, C., and Cubas, P. (2007). Arabidopsis BRANCHED1 acts as an integrator of branching signals within axillary buds. Plant Cell 19, 458-472. doi: 10.1105/tpc.106. 048934

Aukerman, M. J., and Sakai, H. (2003). Regulation of flowering time and floral organ identity by a MicroRNA and its APETALA2-like target genes. Plant Cell 15, 2730-2741. doi: 10.1105/tpc.016238

Busch, A., and Zachgo, S. (2007). Control of corolla monosymmetry in the Brassicaceae Iberis amara. Proc. Natl. Acad. Sci. U.S.A. 104, 16714-16719. doi: 10.1073/pnas.0705338104

Chung, B. Y., Simons, C., Firth, A. E., Brown, C. M., and Hellens, R. P. (2006). Effect of 5' UTR introns on gene expression in Arabidopsis thaliana. BMC Genomics 7:120. doi: 10.1186/1471-2164-7-120

Costa, M. M., Fox, S., Hanna, A. I., Baxter, C., and Coen, E. (2005). Evolution of regulatory interactions controlling floral asymmetry. Development 132, 50935101. doi: 10.1242/dev.02085

Cubas, P., Lauter, N., Doebley, J., and Coen, E. (1999). The TCP domain: a motif found in proteins regulating plant growth and development. Plant J. 18, 215-222. doi: 10.1046/j.1365-313X.1999.00444.X

Efroni, I., Blum, E., Goldshmidt, A., and Eshed, Y. (2008). A protracted and dynamic maturation schedule underlies Arabidopsis leaf development. Plant Cell 20, 2293-2306. doi: 10.1105/tpc.107.057521

Finn, R. D., Clements, J., and Eddy, S. R. (2011). HMMER web server: interactive sequence similarity searching. Nucleic Acids Res. 39, W29-W37. doi: $10.1093 /$ nar/gkr367 interactions take place between different types of PmTCPs, and how TCP-target genes are regulated. Further study is needed to answer these questions and provide an improved understanding of the regulatory network related to key TCP genes.

\section{AUTHOR CONTRIBUTIONS}

$\mathrm{YZ}$ and ZX contributed equally to this work. YZ, ZX, and QZ designed the whole experiments. $Y Z$ wrote the manuscript. YZ, ZX, KZ, WY, TC, and JW analyzed the data. All authors read and approved the final manuscript.

\section{FUNDING}

The research was supported by National Natural Science Foundation of China (Grant No. 31471906), Ministry of Science and Technology (2013AA102607), Special Fund for Beijing Common Construction Project.

\section{ACKNOWLEDGMENT}

We are grateful to Hudson Berkhouse (Texas A\&M University) for improving the manuscript.

\section{SUPPLEMENTARY MATERIAL}

The Supplementary Material for this article can be found online at: http://journal.frontiersin.org/article/10.3389/fpls.2016.01301

Galego, L., and Almeida, J. (2002). Role of DIVARICATA in the control of dorsoventral asymmetry in Antirrhinum flowers. Genes Dev. 16, 880-891. doi: $10.1101 /$ gad.221002

Gaudin, V., Lunness, P. A., Fobert, P. R., Towers, M., Riou-Khamlichi, C., Murray, J. A., et al. (2000). The expression of D-cyclin genes defines distinct developmental zones in snapdragon apical meristems and is locally regulated by the Cycloidea gene. Plant Physiol. 122, 1137-1148. doi: 10.1104/pp.122.4.1137

Giraud, E., Ng, S., Carrie, C., Duncan, O., Low, J., Lee, C. P., et al. (2010). TCP transcription factors link the regulation of genes encoding mitochondrial proteins with the circadian clock in Arabidopsis thaliana. Plant Cell 22, 39213934. doi: 10.1105/tpc.110.074518

Herve, C., Dabos, P., Bardet, C., Jauneau, A., Auriac, M. C., Ramboer, A., et al. (2009). In vivo interference with AtTCP20 function induces severe plant growth alterations and deregulates the expression of many genes important for development. Plant Physiol. 149, 1462-1477. doi: 10.1104/pp.108.126136

Hong, Y., and Jackson, S. (2015). Floral induction and flower formation-the role and potential applications of miRNAs. Plant Biotechnol. J. 13, 282-292. doi: 10.1111/pbi. 12340

Howarth, D. G., and Donoghue, M. J. (2006). Phylogenetic analysis of the "ECE" (CYC/TB1) clade reveals duplications predating the core eudicots. Proc. Natl. Acad. Sci. U.S.A. 103, 9101-9106. doi: 10.1073/pnas.0602827103

Hubbard, L., McSteen, P., Doebley, J., and Hake, S. (2002). Expression patterns and mutant phenotype of teosinte branched 1 correlate with growth suppression in maize and teosinte. Genetics 162, 1927-1935.

Kim, M., Cui, M. L., Cubas, P., Gillies, A., Lee, K., Chapman, M. A., et al. (2008). Regulatory genes control a key morphological and ecological trait transferred between species. Science 322, 1116-1119. doi: 10.1126/science.1164371 
Kosugi, S., and Ohashi, Y. (1997). PCF1 and PCF2 specifically bind to cis elements in the rice proliferating cell nuclear antigen gene. Plant Cell 9, 1607-1619. doi: 10.1105/tpc.9.9.1607

Koyama, T., Furutani, M., Tasaka, M., and Ohme-Takagi, M. (2007). TCP transcription factors control the morphology of shoot lateral organs via negative regulation of the expression of boundary-specific genes in Arabidopsis. Plant Cell 19, 473-484. doi: 10.1105/tpc.106.044792

Koyama, T., Mitsuda, N., Seki, M., Shinozaki, K., and Ohme-Takagi, M. (2010). TCP transcription factors regulate the activities of ASYMMETRIC LEAVES1 and miR164, as well as the auxin response, during differentiation of leaves in Arabidopsis. Plant Cell 22, 3574-3588. doi: 10.1105/tpc.110.07 5598

Larkin, M. A., Blackshields, G., Brown, N. P., Chenna, R., McGettigan, P. A., McWilliam, H., et al. (2007). Clustal W and Clustal X version 2.0. Bioinformatics 23, 2947-2948. doi: 10.1093/bioinformatics/btm404

Li, C., Potuschak, T., Colon-Carmona, A., Gutierrez, R. A., and Doerner, P. (2005). Arabidopsis TCP20 links regulation of growth and cell division control pathways. Proc. Natl. Acad. Sci. U.S.A. 102, 12978-12983. doi: 10.1073/pnas.0504039102

Manassero, N. G., Viola, I. L., Welchen, E., and Gonzalez, D. H. (2013). TCP transcription factors: architectures of plant form. Biomol. Concepts 4, 111-127. doi: 10.1515/bmc-2012-0051

Martin-Trillo, M., and Cubas, P. (2010). TCP genes: a family snapshot ten years later. Trends Plant Sci. 15, 31-39. doi: 10.1016/j.tplants.2009.11.003

Meyers, B. C., Axtell, M. J., Bartel, B., Bartel, D. P., Baulcombe, D., Bowman, J. L., et al. (2008). Criteria for annotation of plant MicroRNAs. Plant Cell 20, 3186-3190. doi: $10.1105 /$ tpc. 108.064311

Nag, A., King, S., and Jack, T. (2009). miR319a targeting of TCP4 is critical for petal growth and development in Arabidopsis. Proc. Natl. Acad. Sci. U.S.A. 106, 22534-22539. doi: 10.1073/pnas.0908718106

Nath, U., Crawford, B. C., Carpenter, R., and Coen, E. (2003). Genetic control of surface curvature. Science 299, 1404-1407. doi: 10.1126/science.10 79354

Ori, N., Cohen, A. R., Etzioni, A., Brand, A., Yanai, O., Shleizer, S., et al. (2007). Regulation of LANCEOLATE by miR319 is required for compoundleaf development in tomato. Nat. Genet. 39, 787-791. doi: 10.1038/ng 2036

Palatnik, J. F., Allen, E., Wu, X., Schommer, C., Schwab, R., Carrington, J. C., et al. (2003). Control of leaf morphogenesis by microRNAs. Nature 425, 257-263. doi: $10.1038 /$ nature 01958

Palatnik, J. F., Wollmann, H., Schommer, C., Schwab, R., Boisbouvier, J., Rodriguez, R., et al. (2007). Sequence and expression differences underlie functional specialization of Arabidopsis microRNAs miR159 and miR319. Dev. Cell 13, 115-125. doi: 10.1016/j.devcel.2007.04.012

Parapunova, V., Busscher, M., Busscher-Lange, J., Lammers, M., Karlova, R., Bovy, A. G., et al. (2014). Identification, cloning and characterization of the tomato TCP transcription factor family. BMC Plant Biol. 14:157. doi: 10.1186/14712229-14-157

Poza-Carrion, C., Aguilar-Martinez, J. A., and Cubas, P. (2007). Role of TCP gene BRANCHED1 in the control of shoot branching in Arabidopsis. Plant Signal. Behav. 2, 551-552. doi: 10.4161/psb.2.6.4811

Rhoades, M. W., Reinhart, B. J., Lim, L. P., Burge, C. B., Bartel, B., and Bartel, D. P. (2002). Prediction of plant microRNA targets. Cell 110, 513-520. doi: 10.1016/S0092-8674(02)00863-2

Sarvepalli, K., and Nath, U. (2011). Hyper-activation of the TCP4 transcription factor in Arabidopsis thaliana accelerates multiple aspects of plant maturation. Plant J. 67, 595-607. doi: 10.1111/j.1365-313X.2011. 04616.x
Schommer, C., Bresso, E., Spinelli, S., and Palatnik, J. (2012). "Role of MicroRNA miR319 in plant development," in MicroRNAs in Plant Development and Stress Responses, ed. R. Sunkar (Heidelberg: Springer), 29-47.

Schommer, C., Debernardi, J. M., Bresso, E. G., Rodriguez, R. E., and Palatnik, J. F. (2014). Repression of cell proliferation by miR319-regulated TCP4. Mol. Plant 7, 1533-1544. doi: 10.1093/mp/ssu084

Schommer, C., Palatnik, J. F., Aggarwal, P., Chetelat, A., Cubas, P., Farmer, E. E., et al. (2008). Control of jasmonate biosynthesis and senescence by miR319 targets. PLoS Biol. 6:e230. doi: 10.1371/journal.pbio.0060230

Sturn, A., Quackenbush, J., and Trajanoski, Z. (2002). Genesis: cluster analysis of microarray data. Bioinformatics 18, 207-208. doi: 10.1093/bioinformatics/18.1.207

Takeda, T., Amano, K., Ohto, M. A., Nakamura, K., Sato, S., Kato, T., et al. (2006). RNA interference of the Arabidopsis putative transcription factor TCP16 gene results in abortion of early pollen development. Plant Mol. Biol. 61, 165-177. doi: 10.1007/s11103-006-6265-9

Viola, I. L., Uberti Manassero, N. G., Ripoll, R., and Gonzalez, D. H. (2011). The Arabidopsis class I TCP transcription factor AtTCP11 is a developmental regulator with distinct DNA-binding properties due to the presence of a threonine residue at position 15 of the TCP domain. Biochem. J. 435, 143-155. doi: 10.1042/BJ20101019

Wang, T., Hao, R., Pan, H., Cheng, T., and Zhang, Q. (2014a). Selection of suitable reference genes for quantitative Real-time polymerase chain reaction in Prunus mume during flowering stages and under different abiotic stress conditions. J. Am. Soc. Hortic. Sci. 139, 113-122.

Wang, T., Pan, H., Wang, J., Yang, W., Cheng, T., and Zhang, Q. (2014b). Identification and profiling of novel and conserved microRNAs during the flower opening process in Prunus mume via deep sequencing. Mol. Genet. Genomics 289, 169-183. doi: 10.1007/s00438-013-0800-6

Xu, R., Sun, P., Jia, F., Lu, L., Li, Y., Zhang, S., et al. (2014). Genomewide analysis of TCP transcription factor gene family in Malus domestica. Genetics 93, 733-746. doi: 10.1007/s12041-014-0446-0

Xu, Z., Sun, L., Zhou, Y., Yang, W., Cheng, T., Wang, J., et al. (2015). Identification and expression analysis of the SQUAMOSA promoter-binding protein (SBP)box gene family in Prunus mume. Mol. Genet. Genomics 290, 1701-1715. doi: 10.1007/s00438-015-1029-3

Xu, Z., Zhang, Q., Sun, L., Du, D., Cheng, T., Pan, H., et al. (2014). Genomewide identification, characterisation and expression analysis of the MADSbox gene family in Prunus mume. Mol. Genet. Genomics 289, 903-920. doi: 10.1007/s00438-014-0863-z

Yang, X., Pang, H. B., Liu, B. L., Qiu, Z. J., Gao, Q., Wei, L., et al. (2012). Evolution of double positive autoregulatory feedback loops in CYCLOIDEA2 clade genes is associated with the origin of floral zygomorphy. Plant Cell 24, 1834-1847. doi: 10.1105/tpc.112.099457

Zhang, Q., Chen, W., Sun, L., Zhao, F., Huang, B., Yang, W., et al. (2012). The genome of Prunus mume. Nat. Commun. 3:1318. doi: 10.1038/ncomms2290

Conflict of Interest Statement: The authors declare that the research was conducted in the absence of any commercial or financial relationships that could be construed as a potential conflict of interest.

Copyright ( 2016 Zhou, Xu, Zhao, Yang, Cheng, Wang and Zhang. This is an open-access article distributed under the terms of the Creative Commons Attribution License (CC BY). The use, distribution or reproduction in other forums is permitted, provided the original author(s) or licensor are credited and that the original publication in this journal is cited, in accordance with accepted academic practice. No use, distribution or reproduction is permitted which does not comply with these terms. 